\title{
人类红细胞胞内束缚水的EPC与DSC方法比 较研究及其应用*
}

\author{
赵 刚 ${ }^{* *}$ 何立群 ${ }^{* * *}$ 郭晓婕刘 忠
}

(中国科学技术大学热科学和能源工程系, 合肥 230027) (合肥红十字会中心血站, 合肥 230031)

\section{罗大为 高大勇}

(Department of Mechanical Engineering, University of Kentucky, Lexington, KY 40506-0108, USA)

摘要使用新渘的差示扫描量热仪方法测定了人类红细胞从 $0^{\circ} \mathrm{C}$ 冷冻至 $-40^{\circ} \mathrm{C}$ 这一过程终结时的体积值, 为等渗体积的 $53 \%$. 使用一种新型的颗粒粒度及计数 分析仪 (Multisizer ${ }^{\mathrm{TM}} 3$, Beckman Coulter Inc, USA)测定了人类红细胞在各种非等 渗溶液中的平衡体积, 溶液渗透压增加至 $3186 \mathrm{mOsm}\left(\mathrm{mol} \cdot \mathrm{kg}_{\text {水 }}^{-1}\right)$, 红细胞的最 终体积值为等渗体积的 $57 \%$. 两种实验方法均表明大约 $34 \%$ 40\%的红细胞胞内 水被束缚在细胞内，不能参与渗透性迁移。实验结果和文献结论“至少 $20 \%$ ～ $32 \%$ 的等渗胞内水被保留在红细胞内”相符. 基于这一实验结果, 改进了传统的细胞 冷冻模型, 并使用改进后的模型预测冷冻过程中红细胞在不同降温速率下的体 积变化过程。随后对这一结果在细胞低温冷冻保存和冻干保存中的应用进行了 探讨.

关键词 差示扫描量热仪 颗粒粒度及计数分析仪 细胞体积变化 红细胞

在红十字会输血中心或医院等部门，血液的保存方法起着一个及其重要的 作用. 目前, 大多数血液制品都是保存在 $4^{\circ} \mathrm{C}$ 或者 $-80^{\circ} \mathrm{C}$ 冰箱内, 少数保存在成本 昂贵的 $-196^{\circ} \mathrm{C}$ 液氮生物容器中. 这类血液保存方法依赖于设备的数量及容量. 尽 管血站等输血部门可以满足日常血液制品的需求，但却无法保证自然或人为灾

2003-09-16 收稿, 2003-12-11 收修改稿

*中国科学院“百人计划”(2000-2003)、国家自然科学基金(批准号：50106016)和安徽省自然科学基金 (批准号: 00047520,03043717)资助项目

**Email: zhaogang@ustc.edu.cn

*** Email: heliqun@ustc.edu.cn 
难造成的紧急大量用血, 因而世界范围内已掀起一个寻求更有效的红细胞保存 技术的热潮. 冻干红细胞具有较好的稳定性与宽松的保存条件等优点，因而作为 一个很有前景的备选方法，冻干技术已经被广泛研究 ${ }^{[1,2]}{ }^{11}$. 在冻干过程中，一些 参数的选择对冻干产品的质量控制至关重要，其中一个参数为红细胞在经历干 燥过程前胞内含水量, 换而言之, 即冷冻过程结束后红细胞胞内束缚水(一个不 确切的叫法一一不可冻结水)的含量. 在干燥过程中, 样品中固态水(冰)首先被蒸 发(初级干燥), 随后进行更高温度下的二级干燥过程(低于红细胞塌陷温度). 为 维持细胞生物活性，大约 5\% 10\%的胞内水必须保留. 为及时停止冻干过程，准 确测定二级干燥前红细胞胞内束缚水的含量非常重要. 尽管该束缚水的含量可 通过核磁共振(NMR)方法测定 ${ }^{[3]}$, 使用差示扫描量热仪(DSC)却是一种更方便快 捷的新方法，该方法已在低温生物学和食品工业等领域广泛应用 $[4,5]$. 最近 Devireddy和Bischof等人发明了一种新颖的DSC方法，可测定冷冻过程中细胞体积 的动态变化过程, 并且给出了一套理论假说, 成功解释了这一低温下的现象 ${ }^{[5]}$. 以 此为理论基础，Luo等人提出了一种基于 DSC 的更简单快捷的分析和实验方法, 可测定细胞在经历了慢速冷冻过程后胞内束缚水的含量 ${ }^{[6]}$. 这是使用DSC方法来 优化冻干过程实验步骤设计的起点, 并且该方法可以连续动态地测定样品内束 缚水的含量. 本文给出了使用颗粒粒度及计数分析仪(EPC)来测定红细胞胞内束 缚水的新方法, 使用一种新型EPC(Multisizer ${ }^{\mathrm{TM}}$ III, Beckman Coulter Inc, USA)测 定了人类红细胞在各种不同质量渗摩尔浓度的 $\mathrm{NaCl}$ 溶液中的最终平衡体积, 根 据该实验结果即可间接模拟慢速冷冻过程中细胞体积的变化过程，直至冷冻过 程结束. 该EPC方法被用来测定红细胞胞内束缚水的含量, 其结果与DSC方法相 符. 两种方法的实验结果均有益于冻干过程的设计与优化. 同时, 本研究指出, 由于红细胞胞内水的存在，使用传统的细胞冷冻模型来预测红细胞在冷冻过程 中的体积变化 (失水过程)时, 必须做出修正, 并且给出了修正前后的模型预测与 实验结果的比较.

\section{DSC 和 EPC 方法的实验原理}

\subsection{DSC 方法的理论依据}

假定（） 慢速冷冻条件下，不出现胞内冰; (ii) 在冷冻过程中，胞内水一旦 透过细胞膜扩散至胞外溶液，立即全部结冰; (iii) 细胞悬浮液内所有组分(包括 溶液和细胞本身)具有相等的密度. 在以上假定下, 从某个起始温度冷冻至任意 终结温度, 单位质量细胞悬浮液的热量释放可以用以下公式描述 ${ }^{[5,6]}$ :

$$
q_{\mathrm{f}}=K_{\mathrm{f}} \cdot \alpha+B_{\mathrm{f}},
$$

1) James P. Dolan. Use of volumetric heating to improve heat transfer during vial freeze-drying. Dissertation for Ph D, Virginia Polytechnic Institute and State University, Blacksburg, Virginia, 1998 


$$
\begin{gathered}
K_{\mathrm{f}}=\left(\frac{V_{0}-V_{\mathrm{f}}}{V_{0}}\right) \cdot \Delta H_{\mathrm{f}}-q_{\mathrm{ec}, \mathrm{f}}, \\
B_{\mathrm{f}}=q_{\mathrm{ec}, \mathrm{f}} .
\end{gathered}
$$

由方程(1) (3), 可得到以下方程, 该方程即可用来计算在任意冷冻终结温度细胞 的体积值:

$$
V_{\mathrm{f}}=V_{0}\left(1-\frac{K_{\mathrm{f}}+B_{\mathrm{f}}}{\Delta H_{\mathrm{f}}}\right),
$$

其中, $q_{\mathrm{f}}$ 是在冷冻过程结束时单位质量细胞悬浮液的总热量释放, 该热量释放是 由于细胞外以及细胞内扩散至细胞外的水冻结导致的; $q_{\mathrm{ec}, \mathrm{f}}$ 是细胞外悬浮液在上 述冷冻过程中的热量释放 (不包括细胞内的水扩散至细胞外后结冰产生的热量 释放); $\alpha$ 是细胞悬浮液的压积; $V_{\mathrm{f}}$ 是冷冻过程结束后最终的细胞体积; $V_{0}$ 是初始的 等渗细胞体积; $\Delta H_{\mathrm{f}}$ 是单位质量纯冰的融解热.

从方程(1)可以看出, $q_{\mathrm{f}}$ 是压积 $\alpha$ 的线性函数. 如果通过实验测定具有不同压 积 $\alpha$ 的细胞悬浮液总的热量释放 $q_{\mathrm{f}}$, 那么斜率 $K_{\mathrm{f}}$ 和截距 $B_{\mathrm{f}}$ 可通过线性拟合实验数 据得到. 将其带入方程(4), 可得到冷冻过程结束后细胞的最终体积 $V_{\mathrm{f}}$.

\subsection{EPC 方法的基本原理}

一种新型的 $\mathrm{EPC}$ 被用来测定人类红细胞在不同质量渗摩尔浓度的 $\mathrm{NaCl}$ 溶液 中的平衡体积. $\mathrm{EPC}$ 方法的基本原理是电子感应区技术, 图 1 是这一原理的示意 图(Multisizer 3 Operator's Manual, PN 8321681 Rev. B). 该方法的理论依据是测定 粒子通过感应区时两个电极之间电阻的变 化. 在真空原的吸力下, 粒子悬浮液透过 小孔管的小孔从管外被抽到馆内, 每一个 粒子均取代与之具有相同体积的电解液. 体积的取代伴随着电压的脉冲. 每一个脉 冲的高度正比于粒子的体积值, 而与粒子 的形状无关. EPC 随机软件(Multisizer 3 V3.51) 可以给出粒子数、粒子体积和粒子表 面积的数量分布. 图 2 为一典型的测量结 果, 人类红细胞在质量渗摩尔浓度为 1349 $\mathrm{mOsm}$ 的 $\mathrm{NaCl}$ 溶液中的平衡体积的分布.

人类红细胞 $(\mathrm{RBC})$ 在不同质量渗摩尔 浓度溶液中平衡体积的变化可以使用 Boyle-van't Hoff关系描述, 即 $V=M_{0} / M \times$

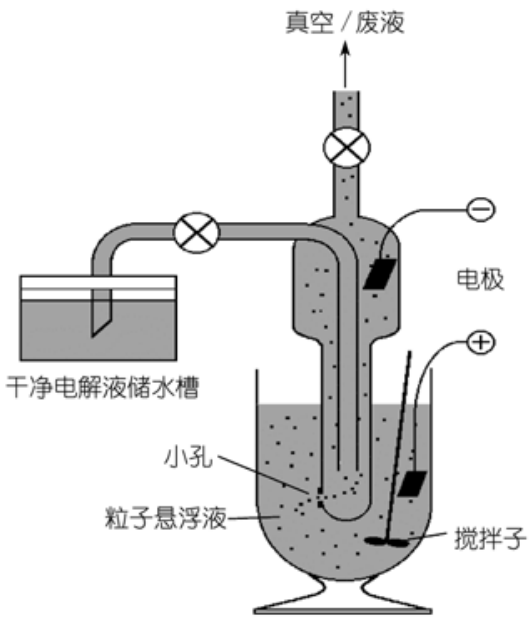

图 1 Multisizer $^{\mathrm{TM}}$ III型电子粒子 计数器的原理图 


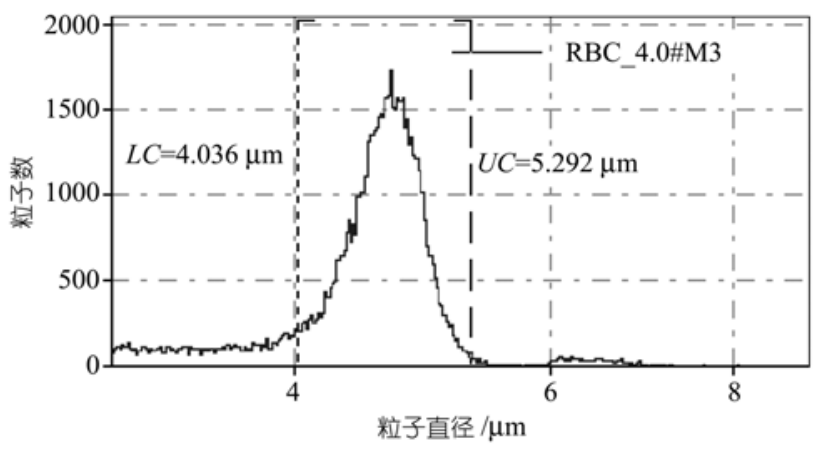

图 2 超渗溶液 $1349 \mathrm{mOsm}$ 中典型的红细胞粒度分布 $L C$ 和 $U C$ 之间粒子数为 56860

$\left(V_{0}-V_{\mathrm{b}}\right)+V_{\mathrm{b}}$. 其中, $V_{0}$ 是在等渗质量渗摩尔浓度 $M_{0}$ 下的细胞体积, $V$ 是细胞在质量 渗摩尔浓度为 $M$ 的溶液中的平衡体积, $V_{\mathrm{b}}$ 是细胞内非渗透性体积 ${ }^{[7]}$. 本研究中, 通 过控制 $\mathrm{NaCl}$ 溶液的浓度控制 $M$ 的值，即间接控制溶液的质量渗摩尔浓度，然后使 用EPC测定人类RBC在不同溶液中的体积值. 从Boyle-van't Hoff关系式可以看出, 如果 $M$ 增加至无限大，则细胞体积趋于 $V_{\mathrm{b}}$.

\section{2 材料和方法}

\section{1 红细胞悬液的制备}

从当地红十字会中心血站获取人类红细胞 (安徽合肥红十字会中心血站), 在 $12 \mathrm{~h}$ 内开始DSC 实验. 通过离心去除上层血浆, 将浓缩的RBC重新悬浮于经过 超滤(PALL-Gelman Laboratory, Super-200, 0.2 $\mu \mathrm{m}$ 滤膜)的生理盐水中. 细胞洗涤 的过程: RBC悬浮液在 $390 \mathrm{~g}(2000 \mathrm{r} / \mathrm{m}$ )下离心 $2 \mathrm{~min}$ (Hettich ZENTRIFUGEN, MIKRO 22 型离心机), 去除上清液, $\mathrm{RBC}$ 被重新悬浮于超虑的 $0.9 \% \mathrm{NaCl}$ 溶液中. 重复以上过程 2 3 次. 细胞悬浮液的一份终浓度被控制在 $3 \times 10^{8} \mathrm{~mL}^{-1}$, 为 $\mathrm{EPC}$ 实 验准备. 另一份被制备成具有 5 个不同压积 $(0,0.35,0.45,0.55,0.62)$ 的样品(离心 后，通过添加不同体积的生理盐水至浓缩的RBC中制备; 依次从 5 个样品中取样 置于压积管中在 $585 \mathrm{~g}(3000 \mathrm{r} / \mathrm{m})$ 下离心 $30 \mathrm{~min}$ 测定压积 $)$ ，为DSC实验准备.

\subsection{DSC 实验步骤}

\subsubsection{DSC 的校正}

使用癸烷和铟两种物质的熔点(癸烷, $\mathrm{C}_{10} \mathrm{H}_{22},-29.7^{\circ} \mathrm{C}$ ，纯度 $99 \%$; 铟, 156.7 ${ }^{\circ} \mathrm{C}$, 纯度 99\%)校正DSC (Pyris-1, Perkin-Elmer Corporation, Norwalk, CT) 的温度. 使用铟的融解潜热 $(28.45 \mathrm{~J} / \mathrm{g})$ 校正DSC的转变焓. 仪器被仔细校正 $2 \sim 3$ 次. 为最 小化仪器中样品的热延迟, 选择较少的样品质量 (12 $\mu \mathrm{L}$, 通常 9 12 mg)均匀地 
放于DSC液体血底, 同时选择比较慢的降温速率 $\left(5^{\circ} \mathrm{C} / \mathrm{min}\right)^{[6]}$.

\subsection{2 总热量释放的测量}

以 $5^{\circ} \mathrm{C} / \mathrm{min}$ 冷却初始平衡在 $4^{\circ} \mathrm{C}$ 的人类 $\mathrm{RBC}$ 悬浮液(每个样品中加入约 $0.1 \mathrm{mg}$ 的Pseudomonas Syringae成核菌), 直至溶液中出现胞外冰(在DSC的温度记录图上 出现一个负的放热峰, 通常在 $-3.5^{\circ} \mathrm{C}$ 左右 $)$. 然后样品被再次平衡在 $-0.53^{\circ} \mathrm{C}\left(T_{\mathrm{ph}}\right.$, $0.9 \%$ 的 $\mathrm{NaCl}$ 溶液的平衡凝固点 $) 4 \mathrm{~min}$. 随后再次以 $5^{\circ} \mathrm{C} / \mathrm{min}$ 从 $T_{\mathrm{ph}}$ 冷却至 $T_{\mathrm{f}}(-0.53 \sim$ $\left.-40^{\circ} \mathrm{C}\right)$. 使用Pyris-1 DSC软件计算冷冻过程的热量释放, 计算过程中均从 $T_{\mathrm{ph}}$ 至 $-25^{\circ} \mathrm{C}$ 取 sigmoidal基线. 每一个样品重复测量 3 次.

\subsection{EPC 实验步骤}

\subsection{1 不同质量渗摩尔浓度的 $\mathrm{NaCl}$ 溶液的制备}

将不同质量的 $\mathrm{NaCl}$ 溶解于使用 KFLOW-R050ACB 水净化系统(KFLOW Water System CO, LTD)制备的去离子水中, 经 $0.2 \mu \mathrm{m}$ 的滤膜(PALL-Gelman Laboratory, Super-200)超滤. 制备出不同质量渗摩尔浓度的 $\mathrm{NaCl}$ 溶液(118, 185, $260,302,670,863,1349,1583,1741,2252,2682,2800,3186 \mathrm{mOsm})$, 质量渗摩尔 浓度使用冰点渗透压计(Osmomat 030 Cryoscopic Osmometer, \pm 5 mOsm, German) 测量, 测量前分别使用去离子水 $(0 \mathrm{mOsm})$ 和标准的 $\mathrm{NaCl}$ 溶液 $(300 \mathrm{mOsm})$ 校准仪 器.

\subsection{2 使用 EPC 测定不同质量渗摩尔浓度下 RBC 的平衡体积}

将制备好的具有不同质量渗摩尔浓度的 $\mathrm{NaCl}$ 溶液各取 $50 \mathrm{~mL}$ 分别加入到容 量为 $50 \mathrm{~mL}$ 的烧杯, 编号 1 13, 然后将 $\mathrm{RBC}$ 分别加入上述 13 个烧杯中, 室温下 平衡 $15 \mathrm{~min}$. 每次更换渗透压后均使用标准橡胶粒子(CC Size Standard L10) 校 正 $\mathrm{EPC}$ 的仪器常数. 然后依次测定细胞悬浮液中 $\mathrm{RBC}$ 的平衡体积. 设置软件每 次运行抽取 30000 个细胞, 每一个样品测量 5 次(本实验选择 $70 \mu \mathrm{m}$ 的小孔管). 然 后使用软件的统计功能给出 30000 个 RBCs 的平均体积作为本次的测量值. 5 次测 量的平均体积值被再次取平均作为最终的平均值, 该值即为相应质量渗摩尔浓 度下 $\mathrm{RBCs}$ 的平衡体积值.

\section{3 实验结果}

\subsection{DSC 实验结果}

表 1 列出了 DSC 方法测量所得到的不同压积的 $\mathrm{RBC}$ 悬浮液在冷冻过程终结 后总的热量释放.

图 3 给出了放热量和压积的关系及其线性拟合公式. 通过线性拟合关系式, 可以得到 $B_{\mathrm{f}}=285.08 \mathrm{~J} / \mathrm{g}, K_{\mathrm{f}}=-127.52 \mathrm{~J} / \mathrm{g}$. 
表 1 DSC 方法测量的热量释放(平均值 $\pm \mathrm{SD}, n=3$ )

\begin{tabular}{clc}
\hline DSC 实验 & 压积 $\alpha$ & $q_{\mathrm{f}}$ \\
\hline 1 & 0 & $285.377 \pm 2.477$ \\
2 & 0.35 & $238.778 \pm 4.879$ \\
3 & 0.45 & $229.416 \pm 3.004$ \\
4 & 0.55 & $214.635 \pm 3.534$ \\
5 & 0.62 & $205.996 \pm 3.637$ \\
\hline
\end{tabular}

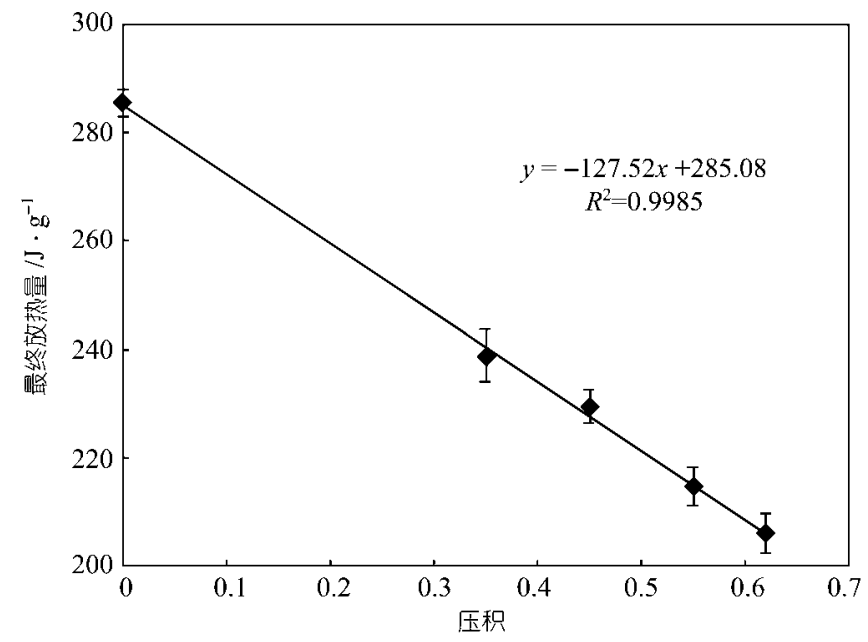

图 3 冷冻 $\left(-0.53 \sim-40^{\circ} \mathrm{C}\right)$ 结束后红细胞悬浮液总的热量释放(平均值 $\pm \mathrm{SD}, n=3$ ) 与压积的关系

将 $B_{\mathrm{f}}$ 和 $K_{\mathrm{f}}$ 带入方程 $(4)$, 假定纯冰的融解热 $\left(\Delta H_{\mathrm{f}}\right)$ 大约为 $333.9 \mathrm{~J} / \mathrm{g}^{[5]}$, 可得 $V_{\mathrm{f}}$ 大 约为 $53 \%$ 的等渗细胞体积.

\subsection{EPC 实验结果}

表 2 给出了 $\mathrm{EPC}$ 方法测定的不同质量渗摩尔浓度下 $\mathrm{RBC}$ 的平衡体积值.

图 4 是平衡 $\mathrm{RBC}$ 的体积与悬浮液的质量渗摩尔浓度的关系. 根据EPC实验的 结果，当质量渗摩尔浓度超过 $2500 \mathrm{mOsm}$ 时， $\mathrm{RBC}$ 的体积基本不再收缩，和文献 结果一致 ${ }^{[8]}$. 红细胞最终的体积约为 $57 \%$ 的等渗体积, 与DSC方法的结果相当. 从以上实验结果可以看出, 水的疏运集在 $118 \sim 1000 \mathrm{mOsm}$ 的质量渗摩尔浓度范 围内. 
表 $2 \mathrm{EPC}$ 方法测定的不同质量渗摩尔浓度下 $\mathrm{RBC}$ 的平衡体积值

\begin{tabular}{ccccccc}
\hline \multirow{2}{*}{ 质量渗摩尔浓度/mOsm } & \multicolumn{5}{c}{ 体积/fL } & \multirow{2}{*}{ 平均体积(平均值 $\pm \mathrm{SD}) / \mathrm{fL}$} \\
\cline { 2 - 5 } & 1 & 2 & 3 & 4 & 5 & \\
\hline 118 & 149.0 & 148.4 & 147.1 & 146.3 & 144.7 & $147.1 \pm 1.7$ \\
185 & 107.2 & 100.6 & 95.82 & 93.71 & 90.98 & $97.66 \pm 6.40$ \\
260 & 80.85 & 78.66 & 75.93 & 76.00 & 74.39 & $77.17 \pm 2.57$ \\
302 & 79.93 & 76.18 & 72.61 & 70.86 & 72.52 & $74.42 \pm 3.64$ \\
670 & 67.14 & 64.86 & 66.36 & 66.78 & 66.28 & $66.28 \pm 0.87$ \\
863 & 58.47 & 59.64 & 59.67 & 59.02 & 59.29 & $59.22 \pm 0.50$ \\
1349 & 49.58 & 49.92 & 49.83 & 49.68 & 49.70 & $49.74 \pm 0.13$ \\
1583 & 52.00 & 51.48 & 51.37 & 51.18 & 50.94 & $51.39 \pm 0.40$ \\
1741 & 48.04 & 48.17 & 47.44 & 47.17 & 47.16 & $47.60 \pm 0.48$ \\
2252 & 43.85 & 46.58 & 46.72 & 41.87 & 42.10 & $44.22 \pm 2.34$ \\
2682 & 41.14 & 41.14 & 41.15 & 43.59 & 43.58 & $42.12 \pm 1.34$ \\
2800 & 43.19 & 43.13 & 43.13 & 43.02 & 43.06 & $43.11 \pm 0.07$ \\
3186 & 42.51 & 42.62 & 42.52 & 42.65 & 42.62 & $42.58 \pm 0.06$ \\
\hline
\end{tabular}

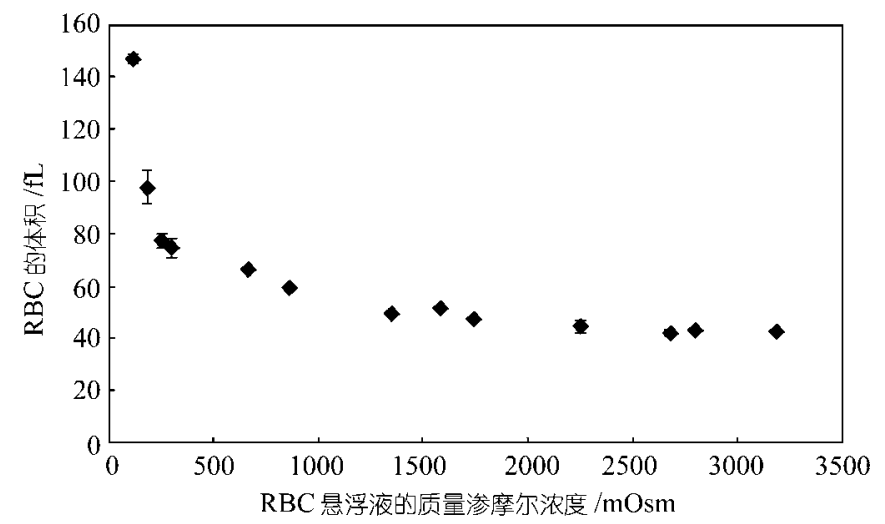

图 4 不同质量渗摩尔浓度下红细胞的平衡体积(平均值 $\pm \mathrm{SD}, n=5$ )

\section{4 结论与讨论}

根据已发表的数据, $\mathrm{RBC}$ 的非渗透性体积约为等渗体积的 $28.3 \%$, 亦即 $V_{\mathrm{b}}=$ $28.3 \% V_{0}$, 文献中分别使用干重法和同位素法测得 ${ }^{[9]}$. 结合本研究的结果, $V_{\mathrm{f}}=$ $53 \sim 57 \% V_{0}$, 可得红细胞内束缚水的含量 $V_{\mathrm{t}, \mathrm{w}}=24.7 \sim 28.7 \% V_{0}$ 或 $34.4 \sim 40 \% V_{0, \mathrm{w}}$, 其 中 $V_{0, \mathrm{w}}$ 是初始等渗条件下 $\mathrm{RBC}$ 内水的体积. 亦即, 仅仅 $60 \sim 65.7 \%$ 的 $\mathrm{RBC}$ 胞内水是 表观可渗透的.

红细胞的体积随质量渗摩尔浓度的变化(无量纲形式)遵循一线性关系 ${ }^{[10]}, V_{\mathrm{b}}$ 一般是将该线性关系外延至质量渗摩尔浓度无限大所得到的细胞体积, 为一理 想情况下的极限值. 通常意义下, $V_{\mathrm{b}}$ 中包括细胞核、细胞器以及它们结合的不可渗 透的水分, 即 $V_{\mathrm{b}}$ 中并不包括自由水. 本研究表明, 在实际冷冻过程中 (以及依靠改 
变胞外溶液质量渗摩尔浓度模拟的平衡冷冻过程), 红细胞在冷冻终结后, 体积 的收缩并不能达到 $V_{\mathrm{b}}$ ，而是停留在一个更大值 $V_{\mathrm{f}}$. 这暗示了 $V_{\mathrm{f}}$ 中包含部分可渗透 水，这部分水与透过细胞膜渗出到胞外的水并无本质区别，只是依然驻留于细胞 内, 最终未能渗出.

伴随外部非渗透性溶质浓度的增加，人类红细胞的行为符合渗透压计，但是 细胞内水的减少小于将红细胞视为理想渗透压计的预测. Ponder常数可以通过从 最终细胞体积 $V_{\mathrm{f}}$ 中减去红细胞的非渗透性体积 $V_{\mathrm{b}}$ 得到，结果为 $0.60 \sim 0.66$.

在 1963 年, Mazur依据热力学基本原理导出了用于描述冷冻过程中细胞体积 变化的数学模型 [11]. 细胞悬浮液冷冻过程中, 由于细胞膜对冰晶生长的阻碍作 用，细胞外溶液中首先出现冰晶 ${ }^{[12]}$. 胞外冰的出现导致细胞内外水的化学势差别, 这一势差驱使胞内水透过细胞膜外流，进一步导致细胞体积变化，该变化可以用 以下方程描述 ${ }^{[11,12]}$ :

$$
\begin{gathered}
\frac{\mathrm{d} V}{\mathrm{~d} T}=\frac{L_{\mathrm{p}} A_{\mathrm{c}} R T}{B v_{\mathrm{w}}}\left[\ln \frac{V-V_{\mathrm{b}}}{V-V_{\mathrm{b}}+\phi_{\mathrm{s}} n_{\mathrm{s}} v_{\mathrm{w}}}-\frac{\Delta H_{\mathrm{f}}}{R}\left(\frac{1}{T_{\mathrm{m} 0}}-\frac{1}{T}\right)\right], \\
L_{\mathrm{p}}=L_{\mathrm{pg}} \exp \left[-\frac{E_{L_{\mathrm{p}}}}{R}\left(\frac{1}{T}-\frac{1}{T_{\mathrm{R}}}\right)\right],
\end{gathered}
$$

其中, $V$ 是细胞体积, $T$ 是绝对温度, $L_{\mathrm{pg}}$ 是在参考温度 $T_{\mathrm{R}}$ 下细胞膜对水的渗透系数, $E_{L_{\mathrm{p}}}$ 是这一渗透过程的表观活化能, $R$ 是气体常数, $B$ 是降温速率, $A_{\mathrm{c}}$ 是对水渗透有 效的膜表面积, $v_{\mathrm{w}}$ 是水的偏摩尔体积, $n_{\mathrm{s}}$ 是细胞内的溶质摩尔数, $V_{\mathrm{b}}$ 是细胞的非渗 透性体积, $\phi_{\mathrm{s}}=2$ 是 $\mathrm{NaCl}$ 在水中的离解常数, $\Delta H_{\mathrm{f}}$ 是纯冰的融解热(为与文献 [6]一 致, 取为 $333.9 \mathrm{~mJ} / \mathrm{mg}), T_{\mathrm{m} 0}$ 是纯冰的平衡凝固点.

在图 5 中, 实心菱形数据点来自 $\mathrm{EPC}$ 实验结果, 这些数据点通过测量 $\mathrm{RBC}$ 在 不同质量渗摩尔浓度溶液中的平衡体积来间接模拟平衡冷冻过程中细胞的体积 变化 ${ }^{[13]}$. 上面的实线为方程(5)中使用 $V_{\mathrm{f}}$ 代替 $V_{\mathrm{b}}$ 后的计算结果. 从图 5 可以看

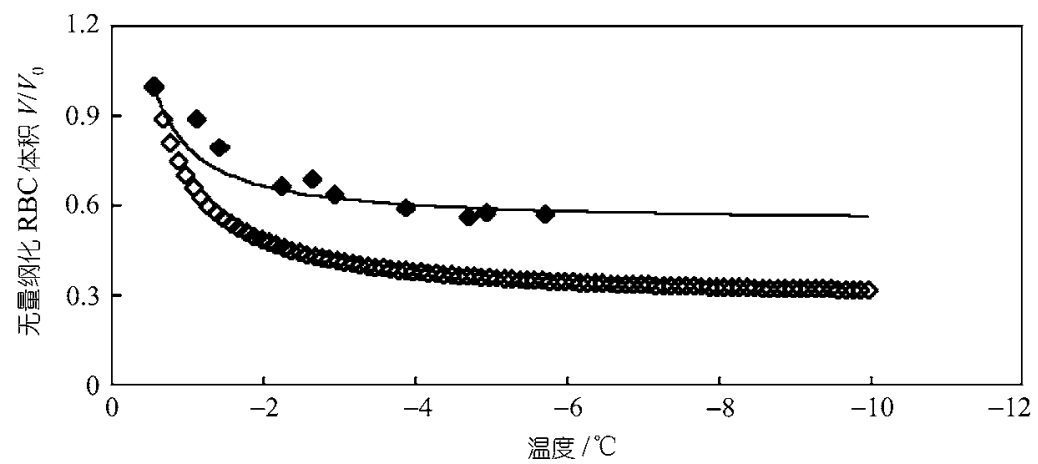

图 5 平衡冷冻过程中 $\mathrm{RBC}$ 体积变化

SCIENCE IN CHINA Ser. E Engineering \& Materials Science 
出, 该结果和 $\mathrm{EPC}$ 实验结果符合的很好, 然而下面的空心菱形点(直接使用 $V_{\mathrm{b}}$ 带入 模型后的计算结果)却和实验结果符合的不够理想. 这里使用平衡冷冻过程这一 特例来探讨模型中该体积的取值对于其计算结果的影响, 避开了Mazur模型中诸 多的参数, 突出了该体积值的直接影响. 这表明, 对于人类红细胞而言, 非渗透 性体积 $V_{\mathrm{b}}$ 不能直接带入传统的细胞冷冻模型中预测实际冷冻过程细胞体积变化, 必须代之以 $V_{\mathrm{f}}$. Levin等人已建立了描述水合对人类红细胞胞内水含量影响的理论 模型 ${ }^{[14]}$, 并使用该模型推测出人 $\mathrm{RBC}$ 内至少有 $16.65 \%(V / V)$ 的等渗胞内水将被束 缚在细胞内而不能参与渗透性迁移. 本研究结果支持该理论预测.

图 6 是降温速率对人 $\mathrm{RBC}$ 在冷冻过程中体积变化过程的影响. 该结果可被 用来优化低温保存过程和冻干过程的程序设计, 并可进一步结合胞内冰晶成核 和生长的模型计算深层冷冻过程中细胞内冰晶的体积份额, 预测细胞经历胞内 冰损伤的程度.

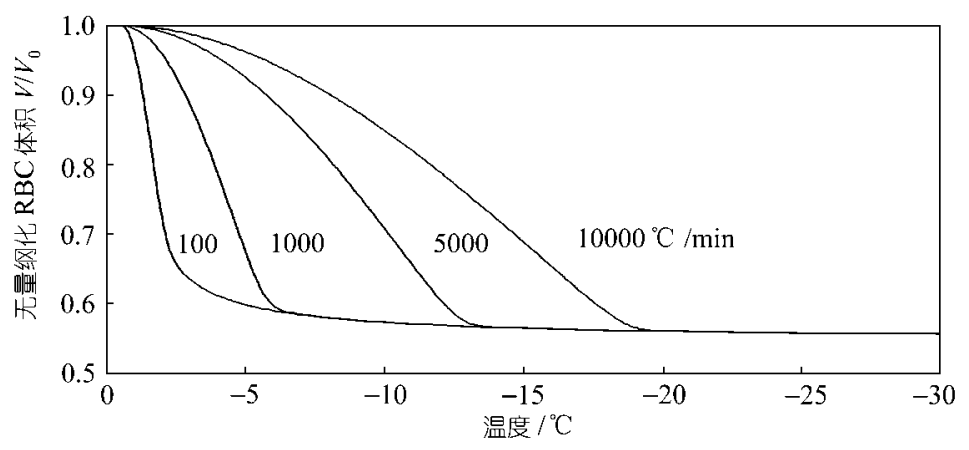

图 6 在不同降温速率下 $\mathrm{RBC}$ 的体积变化

根据本研究结果, 人类红细胞存在部分胞内水不能参与渗透性脱水, 我们称 之为“束缚水”, 因为在冷冻过程中, 随着胞外冰晶的生长导致胞外溶液质量渗摩 尔浓度的增加并不能促使这部分水流出细胞. 束缚水主要以“自由水”(完全可以 参与渗透性流动, 但冷冻过程中被舒服在细胞内而未能外流)的形式存在，同时 可能有小部分以“结合水”(与红细胞内的溶质和蛋白质大分子等水合)的形式存 在. 红细胞低温冷冻保存和冻干保存的成功设计必须充分考虑该 “束缚水” 对于 细胞冷冻响应带来的影响.

\section{参 考 文 献}

1 Rindler V, Luneberger S, Schwindke P, et al. Freeze-drying of red blood cells at ultra-low temperatures. Cryobiology, 1999, 38: 2 15[DOI]

2 Rindler V, Heschel I, Rau G. Freeze-drying of red blood cells: how useful are freeze/thaw experiments for optimization of the cooling rate? Cryobiology, 1999, 39: 228 235[DOI] 
3 Cooke R, Kuntz I D. The properties of water in biological systems. Annu Rev Biophys Bioeng, 1974, 3: 95 126

4 Kenneth D R. Differential scanning calorimetry of nonfreezable water in solute-macromolecule-water system. Journal of Food Science, 1978, 43: 1813 1816

5 Ramachandra V D, Debopam R, Bischof J C. Measurement of water transport during freezing using differential scanning calorimetry. Cryobiology, 1998, 36: 124 155[DOI]

6 Luo D W, Han X, He L Q, et al. Determination of intracellular residual unfrozen water using a DSC approach. Cryo-Letters, 2002, 23: 229 236

7 Jason P A, Julia P, Ingo $\mathrm{H}$, et al. Comparison of optical measurement and electrical measurement techniques for the study of osmotic responses of cell suspensions. Cryo-Letters, 1999, 20: 315 324

8 John F, Woolgar A E. Human red cells under hypertonic conditions: a model system for investigating freezing damage, I. Sodium chloride. Cryobiology, 1972, 9: 9 15

9 David S, Victor W S, Solomon A K. Osmotic properties of human red cells. The Journal of General Physiology, 1964, 48: 79 94

10 华泽钊, 任禾盛. 低温生物医学技术. 北京: 科学出版社, 1994. 34 35

11 Mazur P. Kinetics of water loss from cells at subzero temperatures and the likehood of intracellular freezing. J Gen Physiol, 1963, 47: 347 369

12 Mazur P. Freezing of living cells: mechanisms and implications. Am J Physiol, 1984, 143: C125 142

13 赵 刚, 何立群, 王沛涛, 等. 平衡冷冻过程中细胞体积的确定. 科学通报, 2003, 48(10): 1019 1022[摘要][PDF]

14 Levin R L, Cravalho E G, Huggins C E. Effect of hydration on the water content of human erythrocytes. Biophysical Journal, 1976, 16: 1411 1426 